\title{
Usefulness of C-Reactive Protein as a Marker for Prediction of Future Coronary Events in the Asian Indian Population: Indian Atherosclerosis Research Study
}

\author{
Veena S. Rao, ${ }^{1}$ Natesha B. Kadarinarasimhiah, ${ }^{1}$ Shibu John, ${ }^{2}$ Sridhara Hebbagodi, ${ }^{2}$ \\ Jayashree Shanker, ${ }^{3}$ and Vijay V. Kakkar ${ }^{4}$ \\ ${ }^{1}$ Tata Proteomics and Coagulation Unit, Thrombosis Research Institute, India, Narayana Hrudayalaya Hospital, \\ 560099 Bangalore, India \\ ${ }^{2}$ Elizabeth and Emmanuel Kaye Bioinformatics and Statistical Unit, Thrombosis Research Institute, 560099 Bangalore, India \\ ${ }^{3}$ Garry and Mary Weston Functional Genomics Unit, Thrombosis Research Institute, 560099 Bangalore, India \\ ${ }^{4}$ Thrombosis Research Institute, Manresa Road, Chelsea, SW36LR London, UK \\ Correspondence should be addressed to Veena S. Rao, veenasrao@triindia.org.in
}

Received 23 February 2010; Accepted 31 May 2010

Academic Editor: John A. Kern

Copyright ( $) 2010$ Veena S. Rao et al. This is an open access article distributed under the Creative Commons Attribution License, which permits unrestricted use, distribution, and reproduction in any medium, provided the original work is properly cited.

Inflammation plays a pivotal role in all stages of atherosclerosis. Numerous inflammatory, lipid, and cytokines markers have been associated with coronary artery disease (CAD) risk but data directly comparing their predictive value are limited. Studies were carried to elucidate the role of high-sensitivity C-reactive protein (hsCRP), other inflammatory as well as lipid markers and their associations. Among 1021 subjects, comprising 774 CAD affected members from Indian Atherosclerosis Research Study (IARS), plasma hsCRP levels showed strong correlation with inflammatory markers, namely, IL6 $(r=.373 ; P=<.0001)$, sPLA2 ( $r=.544$; $P=<.0001)$ as also with fibrinogen $(r=.579 ; P=<.0001)$. Levels of hsCRP were higher among subjects affected by CAD who suffered a repeat coronary event as compared to those who remained event free and subjects in the top quartile of hsCRP $(>3.58 \mathrm{mg} / \mathrm{L}$ ) were found to have a fourfold higher risk. In conclusion, hsCRP appears to be an independent predictor of recurrent $\mathrm{CAD}$ events in Asian Indian population.

\section{Introduction}

Inflammation plays a major role in the pathophysiology of atherosclerosis [1-4]. Many novel inflammatory markers have been investigated in an effort to improve the risk predictive ability of future coronary artery disease (CAD) [58]. With the exception of high-sensitivity C-reactive protein (hsCRP), no inflammatory marker evaluated thus far has demonstrated consistent and independent value towards prediction of future CAD risk. High sensitive CRP has been advocated as the most attractive candidate novel biomarker for inclusion in existing risk prediction models, primarily due to its stability, low diurnal variation, and ease of measurement [9]. hsCRP has been accepted to provide additional value to the Framingham risk scoring system devised for future 10yr CAD risk prediction, even in the presence of conventional CAD risk factors [10-13].
CRP is an acute-phase reactant belonging to the pentraxin family. It is almost exclusively produced in the hepatocytes under the control of cytokines [14], although extrahepatic sites of CRP synthesis have been reported [1518]. The major functions of CRP include binding to various ligands on damaged tissue followed by propagation of both anti- and proinflammatory effects [19-21]. Recent evidence indicates that CRP plays an active role in atherosclerosis $[22,23]$. There is general consensus that individuals in the top quartile of CRP have a two- to three-fold higher risk of an incident as well as future coronary event compared to those in the bottom quartile [24]. However, there is ongoing debate on the potential of plasma CRP to predict future coronary risk over and above that of established CAD risk factors. The existing reports are contradictory [6, 12, 24-35]. Significant race-based differences in CRP levels have been reported. Considering the prevalent lacunae in current knowledge, 
more so with respect to the Asian Indian population, this study was designed to assess the value of hsCRP in predicting future $\mathrm{CAD}$ in a cohort of high-risk Indians residing in the Indian subcontinent.

\section{Methods}

The Indian Atherosclerosis Research Study (IARS) is an ongoing, family-based, epidemiological study initiated in 2004 to investigate the prevalent genetic, traditional, and environmental factors associated with $\mathrm{CAD}$ in a cohort of Asian Indians in their home country. Novel biomarker discovery is one of the many specific objectives of this large-scale prospective study. The families in the IARS were enrolled from two cities: Bangalore in south India and Mumbai in western India. Subjects were recruited through a proband treated for CAD and its complications. A proband was eligible for recruitment when he was older than 60 (men) or 65 (women) years at onset of CAD. This was done in order to identify families with early onset CAD. All probands had a positive family history of CAD/CVD. We defined CADaffected individuals as those who had documented evidence of an acute coronary syndrome with or without angiographic evidence of CAD and/or had undergone revascularization procedures. Probands' family members (both affected and unaffected by CVD) were subsequently enrolled into the study, provided they were aged 18 years or above at the time of recruitment. There was no upper age limit for any of the nonproband recruits. Subjects with present or past major illnesses such as cancer, cardiomyopathy, rheumatic heart disease, liver or renal disease and concomitant infection were excluded from the study. All participants gave their written informed consent to participate in the study, which was approved by the local ethics committee.

A detailed case record form containing information on demographics, anthropometry, and medical history of diabetes, hypertension, and CVD was completed for all participants. A general physical examination was performed, along with measurement of vital parameters. Relevant information was obtained by personal interviews with subjects and from medical records. Prevalence of diabetes, hypertension, and CVD was ascertained based on self-report of physician's diagnosis and/or use of prescription medications along with medical records of diagnosis and therapy. Patients were followed up via telephone calls once every year. This followup data was collected during conversation with the patients themselves and all clinical data, including current medical therapy was judiciously recorded.

2.1. Biomarker Assays. All the biomarker assays were performed in accordance to standard protocols at the central research facility of the Thrombosis Research Institute (TRI) at Bangalore. Serum total cholesterol and triglyceride were estimated by standard enzymatic analysis using reagents, standards and controls from Randox Laboratories Ltd. (Antrim, UK). The concentrations of high-density lipoprotein (HDL)-cholesterol were estimated after precipitation of non-HDL fractions with a mixture of $2.4 \mathrm{mmol} / \mathrm{L}$ phosphotungstic acid and $39 \mathrm{mmol} / \mathrm{L}$ magnesium chloride. The concentrations of low-density lipoprotein (LDL)-cholesterol were calculated using the Fridewald formula. Apolipoproteins A1 and B100 (Orion Diagnostics, Espoo, Finland) were estimated by immunoturbidimetry method. Analyses were carried out on a Cobas-Fara II Clinical Chemistry Autoanalyser (F. Hoffmann La Roche Ltd., Basel, Switzerland). Three commercial controls purchased from Randox Laboratories, one from Orion Diagnostica for apolipoproteins and a normal human serum pool (NHP) prepared in-house was run with every batch of assays. The interassay coefficients of variation $(\mathrm{CV})$ for the commercial controls and NHP ranged from $4.9 \%$ to $7.0 \%$ for total cholesterol, $6.1 \%$ to $7.7 \%$ for triglyceride, $7.1 \%$ to $12.2 \%$ for HDL-cholesterol, $3.3 \%$ to $5.2 \%$ for $\operatorname{Lp}(\mathrm{a}), 9.9 \%$ to $14.2 \%$ for apolipoprotein $\mathrm{A} 1$, and $10.7 \%$ to $13.9 \%$ for apolipoprotein B100.

Plasma interleukin (IL)-6 level was measured by enzymelinked immunosorbent assay ( $\mathrm{R} \& \mathrm{D}$ Systems, Minneapolis, USA); the interassay CV for NHP was $4.3 \%$. Plasma hsCRP level was measured using the Roche latex Tina quant kit (Roche Diagnsotics, Basel, Switzerland); the CV of NHP was $7.85 \%$. Levels of secretory phospholipase A2 (sPLA2) were determined using a sandwich immunometric assay (Cayman Corporation, Michigan, USA) and the interassay CV of NHP was 5.37\%. Plasma fibrinogen and FVII:C activity were measured in clotting assays on an Automated Coagulation Analyser (ACL 300, Instrumentation Laboratory, Milano, Italy). The reagents, calibrators, and controls were purchased from IL (Lexington, MA, USA). A commercial control and pooled normal human plasma (NHP) prepared in-house from citrate plasma of 30 healthy volunteers were run with every batch of assay. The interassay $\mathrm{CV}$ of the commercial control and NHP were $5.7 \%$ and $5.9 \%$, respectively, for fibrinogen and $5.9 \%$ and $11.6 \%$, respectively, for FVII:C. The values of fibrinogen and FVII:C for the commercial control were within the pre-assigned ranges in every batch of assay.

2.2. Statistical Analysis. All statistical analyses were performed using SPSS version 12. A $P$-value of less than.05 was considered statistically significant. Variables that showed a skewed distribution were log transformed before carrying out further statistical analysis. For the purpose of clarity, the untransformed mean and standard error of the mean (SEM) are shown in tables. Differences between continuous variables were assessed using Student's $t$-test and those between categorical variables using the chi square test. Analysis of covariance was also used to test for significance after adjusting for potential confounding variables. Odds ratios (ORs) and corresponding 95\% confidence intervals (CIs) were calculated using conditional logistic regression analysis.

\section{Results}

3.1. Baseline Characteristics of Study Population. A total of 518 families comprising 2318 individuals were recruited into Phase I of the IARS. Table 1 describes the baseline clinical and demographic characteristics of the study population in two groups: CAD-affected individuals who have suffered a coronary event and CAD unaffected people at 
TABLE 1: Baseline characteristics of study participants.

\begin{tabular}{|c|c|c|c|}
\hline & CAD-patients $(n=774)$ & No CAD $(n=1544)$ & $P$ \\
\hline \multicolumn{4}{|l|}{ Continuous variables, mean (SD) } \\
\hline Age & $55.93(9.36)$ & $40.37(14.15)$ & $<.0001$ \\
\hline BMI & $25.83(3.85)$ & $25.67(4.9)$ & .418 \\
\hline Systolic blood pressure ( $\mathrm{mmHg}$ ) & $127.57(16.74)$ & $122.18(15.35)$ & $<.0001$ \\
\hline Diastolic blood pressure $(\mathrm{mmHg})$ & $81.53(8.55)$ & $81(9.26)$ & .186 \\
\hline Total cholesterol (mg/dL)* & $155.41(40.79)$ & $178.27(40.33)$ & $<.0001$ \\
\hline Triglycerides $(\mathrm{mg} / \mathrm{dL})^{*}$ & $149.84(68.86)$ & $131.98(85.09)$ & $<.0001$ \\
\hline HDL-cholesterol (mg/dL)* & $38.75(9.06)$ & $42.46(10.6)$ & $<.0001$ \\
\hline LDL-cholesterol (mg/dL)* & $86.27(33.76)$ & $110.18(34.82)$ & $<.0001$ \\
\hline \multicolumn{4}{|l|}{ Categorical variables, $n(\%)$} \\
\hline Statin & $529(69.2)$ & $44(2.9)$ & $<.0001$ \\
\hline Male & $630(81.6)$ & $724(46.9)$ & $<.0001$ \\
\hline Female & $142(18.4)$ & $820(53.1)$ & $<.0001$ \\
\hline Smoking & $\cdots$ & $\cdots$ & $\cdots$ \\
\hline Never & $465(60.5)$ & $1356(88.1)$ & $\cdots$ \\
\hline Ex-smoker & $241(31.4)$ & $61(3.96)$ & $\cdots$ \\
\hline Occasional & $5(0.7)$ & $25(1.62)$ & $\cdots$ \\
\hline Current smoker & $57(7.4)$ & $98(6.36)$ & $\ldots$ \\
\hline \multicolumn{4}{|l|}{ History of } \\
\hline Diabetes & 349 (67.9) & $529(57.8)$ & $<.0001$ \\
\hline Hypertension & $378(73.8)$ & $659(72.0)$ & .37 \\
\hline
\end{tabular}

Probability values for continuous variables are from 2 -sample $t$-tests and for categorical variables from chi square tests.

* Log-transformed distribution.

the time of recruitment. Traditional coronary risk factors, namely, diabetes, hypertension and lipid profile were more prevalent in the affected group. The lower levels of total and LDL-cholesterol noted in the CAD-affected group may be due to the use of statins in over $70 \%$ of affected subjects.

3.2. Comparison of Baseline hsCRP Levels Among CADAffected and Unaffected Subjects. Fasting venous blood samples for biomarker analysis were collected from each one of the 2318 individuals recruited into phase 1 of the IARS. hsCRP levels were assayed in 1021 out of the above 2318 collected samples. The selection of samples for the study was random. Levels of CRP are known to vary across body mass index (BMI) and gender. However, adjustment for both of these variables as also for statins did not reveal a significant difference in the CRP titres between affected and unaffected individuals (Table 2).

Figure 1 categorizes our study population according to the 2003 Joint American Heart Association and Center for Disease Control and Prevention (AHA-CDC) (Ref) guidelines for risk stratification of CAD based on CRP titres as follows: low risk $<1 \mathrm{mg} / \mathrm{L}$, moderate risk $1-3 \mathrm{mg} / \mathrm{L}$, and high risk $>3 \mathrm{mg} / \mathrm{L}$. We found no significant difference in the distribution of subjects in the three risk classes between the CAD-affected and -unaffected subjects $(P=.13)$, indicating that application of AHA-CDC guidelines for CRP classification may not hold true for all populations.

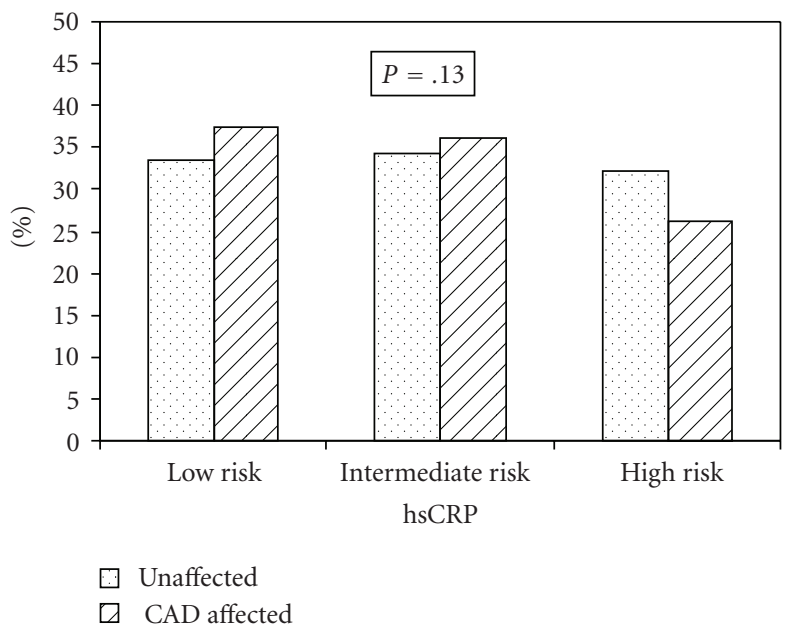

FIGURE 1: Classification of subjects based on high-sensitivity C-reactive protein (hsCRP) levels according to Joint American Heart Association and Centers for Disease Control and Prevention guidelines: low risk $<1 \mathrm{mg} / \mathrm{L}$, moderate risk $1-3 \mathrm{mg} / \mathrm{L}$, and high risk $>3 \mathrm{mg} / \mathrm{L}$. CAD = coronary artery disease.

3.3. Correlation of hsCRP Levels with Inflammatory and Lipid Markers. The unadjusted and adjusted correlation coefficients for hsCRP are shown in Table 3. hsCRP significantly correlated with IL-6, fibrinogen and sPLA2 levels, after 
TABLE 2: Baseline high-sensitivity C-reactive protein (hsCRP) levels (mg/L).

\begin{tabular}{lcccc}
\hline & Male & & \multicolumn{2}{c}{ Female } \\
affected $(n=63)$ & Unaffected $(n=336)$ & $P$ \\
\hline $2.596 \pm 0.26$ & Affected $(n=321)$ & $P$ & $3.303 \pm 0.228$ & $4.658 \pm 0.664$
\end{tabular}

hsCRP test performed in 1021 samples.

*adjusted for body mass index and statin use.

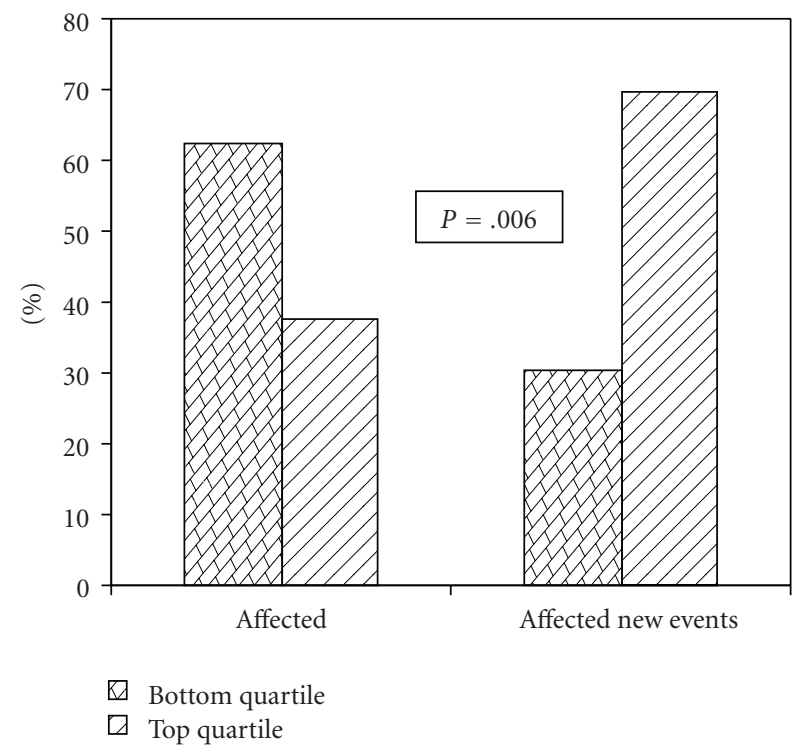

FIGURE 2: Distribution of subjects according to high-sensitivity Creactive protein (hsCRP) quartiles.

adjustment for age, gender, and BMI. There was moderate correlation with lipoproteins.

3.4. hsCRP and Risk of Future CAD Events. The study cohort was periodically followed up over telephone at 1 year intervals. $28.3 \%$ of the original cohort was lost during the follow-up process. Detailed medical information was obtained in the remaining cases (71.7\%). 115 individuals had a new coronary event, irrespective of their baseline disease status (CAD affected or unaffected). CAD-affected subjects who suffered a recurrent event $(n=91)$ had significantly higher levels of hsCRP than affected subjects who remained event free $(3.98 \pm 0.56$ versus $2.9 \pm 0.17(\mathrm{mg} / \mathrm{L}) ; P=.009)$. Over $70 \%$ of CAD-affected individuals who suffered a repeat event were in the top quartile of hsCRP titres (Figure 2).

Logistic regression model was used to determine the ability of hsCRP level to predict future coronary events based on quartile distribution. There was increasingly significant association of CAD risk with increasing quartiles of hsCRP levels. The odds of a recurrent coronary event for CADaffected individuals in the top quartile of hsCRP was about four times that of individuals in the other three quartiles (95\% CI 1.45-9.89; $P=.006$; Model 1, Table 4). After adjusting for traditional risk factors such as hypertension, diabetes, gender, age, and BMI, the significant association between hsCRP and risk of repeat coronary events remained for individuals in the top hsCRP quartile (OR 3.9, 95\%CI 1.41-10.8; $P=.009$; Model 2, Table 4). After additional adjustment for IL-6, although the OR increased to over fourfold, the significance of the association with future CAD events was nominal (OR 4.359, 95\% CI 1.023-18.573; $P=$ .047; Model 3, Table 4). When IL-6 was excluded and lipid variables (total cholesterol, triglycerides, HDL and LDL) were added into the model, the association between the top quartile and risk of repeat CAD event persisted (OR 3.106 95\% CI 1.07-9.014; $P=.037$; Model 4 and 5, Table 4.

\section{Discussion}

The increasing pressure on health resources worldwide, has led to the emphasis on preventive measures in the battle against the global pandemic of CAD $[36,37]$. Indians are arguably at a higher risk of developing CAD than other races and this cannot be explained by traditional risk factors alone [38-42]. Although a multitude of factors have been measured in the blood of subjects with established atherosclerosis, only hsCRP has demonstrated an additive value to the risk assessment of CAD, as endorsed by the Framingham risk score $[9,11,26]$.

The IARS is an ongoing large-scale family-based study that is investigating novel biomarkers that may contribute to enhanced risk of CAD in Asian Indians, thereby enabling the identification of high-risk subjects who may benefit from early intervention. Most published reports of hscRP levels in Asian Indians have been carried out on subjects living outside India [43-46]. There are relatively fewer studies in the Indian population $[47,48]$ that have reported relatively small cohorts. Thus, this is the first study to determine the utility of hsCRP as a marker for risk prediction in Asian Indian subjects with CAD living in their home country.

We did not find a significant difference in baseline levels of hsCRP between CAD affected and unaffected subjects even after adjusting for gender, BMI, and statin therapy. This may possibly be attributed to therapeutic modifications in affected subjects in terms of treatment with inhibitors of arachidonic acid metabolism (aspirin), PPR $\gamma$ antagonists amongst others that are known to possess anti-inflammatory properties in addition to their primary effect. Moreover lifestyle and diet modifications play a significant role in overall risk reduction. In our cohort, over $69 \%$ of the CAD affected subjects were on statin therapy while only $2.9 \%$ were on this drug in the unaffected group. As expected, hsCRP levels displayed significant correlation with inflammatory markers IL-6, sPLA2, fibrinogen, and neopterin (Table 3). Interestingly, when we compared the hsCRP levels in CADaffected subjects who were followed up for 4 years over 
TABLE 3: Correlation of high-sensitivity C-reactive protein (hsCRP) levels with inflammatory markers and lipids.

\begin{tabular}{|c|c|c|c|c|}
\hline \multirow[b]{2}{*}{ Characteristics } & \multicolumn{2}{|c|}{ Unadjusted } & \multicolumn{2}{|c|}{ Adjusted $^{\dagger}$} \\
\hline & Correlation & $P$ & Correlation & $P$ \\
\hline sPLA2 $(\mathrm{pg} / \mathrm{mL})^{*}$ & 0.544 & $<.0001$ & 0.464 & $<.0001$ \\
\hline Interleukin $6(\mathrm{pg} / \mathrm{mL})^{*}$ & 0.373 & $<.0001$ & 0.325 & $<.0001$ \\
\hline Total cholesterol (mg/dL) & 0.156 & $<.0001$ & 0.11 & $<.0001$ \\
\hline $\mathrm{HDL}(\mathrm{mg} / \mathrm{dL})$ & -0.147 & $<.0001$ & -0.163 & $<.0001$ \\
\hline $\mathrm{LDL}(\mathrm{mg} / \mathrm{dL})$ & 0.154 & $<.0001$ & 0.114 & $<.0001$ \\
\hline ApoA1 (g/L) & 0.043 & .168 & 0.029 & .354 \\
\hline ApoB $(g / L)$ & 0.201 & $<.0001$ & 0.17 & $<.0001$ \\
\hline Triglyceride $(\mathrm{mg} / \mathrm{dL})^{*}$ & 0.208 & $<.0001$ & 0.161 & $<.0001$ \\
\hline Fibrinogen $(\mathrm{g} / \mathrm{L})$ & 0.579 & $<.0001$ & 0.522 & $<.0001$ \\
\hline FVII:C (\%) & 0.215 & $<.0001$ & 0.105 & .001 \\
\hline
\end{tabular}

${ }^{*} \log$-transformed distribution.

${ }^{\dagger}$ Partial correlations adjusted for age, gender, body mass index, and smoking status.

sPLA2: secretory phospholipase A2; HDL: high-density lipoprotein; LDL: low-density lipoprotein; ApoA1: apolipoprotein A1; ApoB: apoplipoprotein B100; FVII:C: factor VII coagulant activity.

TABLE 4: Risk of recurrence of coronary artery disease (CAD) events based on high-sensitivity C-reactive protein (hsCRP) quartiles.

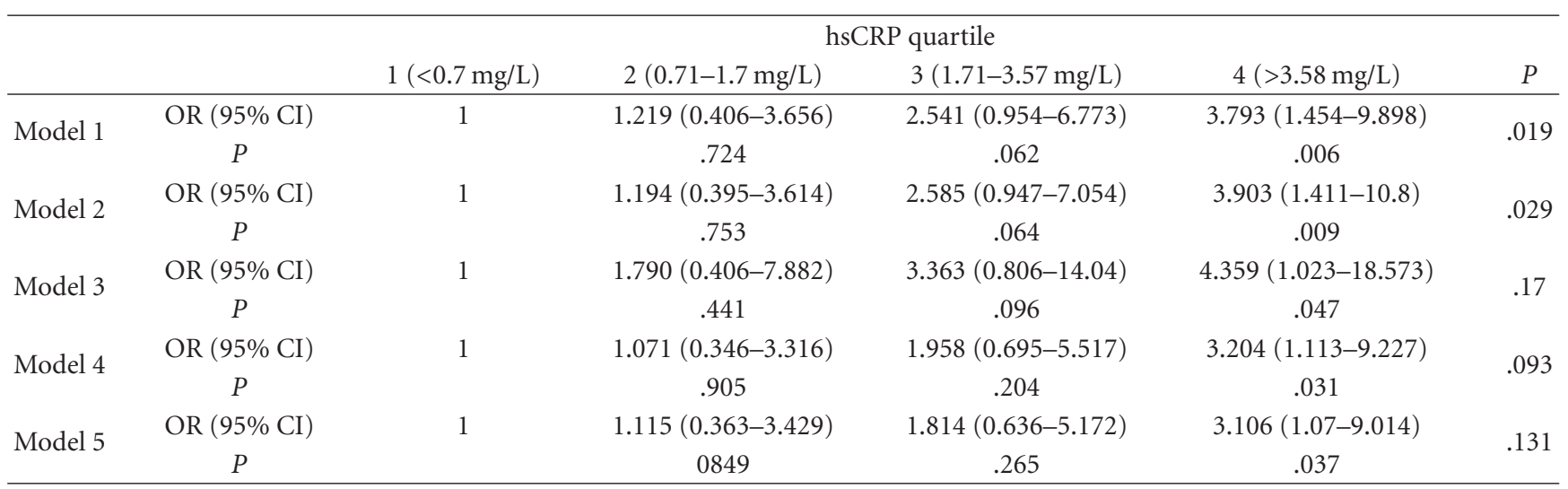

Model 1: Unadjusted.

Model 2: Adjusted for hypertension, diabetes, gender, age, and body mass index.

Model 3: Additional adjustment for interlukin-6*

Model 4: Additional adjustment total cholesterol, high-density lipoprotein (HDL) and triglyceride*.

Model 5: Additional adjustment total cholesterol, HDL, triglyceride* and low-density lipoprotein.

* log-transformed distribution.

OR: odds ratio; CI: confidence interval.

telephone to assess their cardiovascular health, we found that about $70 \%$ of those who developed a recurrent event were in the top hsCRP quartile $(>3.58 \mathrm{mg} / \mathrm{L})$. These individuals have approximately four times higher risk of developing a recurrent coronary event than those in the bottom quartile (OR 3.79, 95\% CI 1.45-9.89; $P=.006$ ). This risk persisted even after adjusting for traditional risk factors such as gender, diabetes, hypertension, BMI, and lipids (Table 4, Model 5), indicating that hsCRP may be an independent predictor of recurrent CAD event in our population. This observation agrees with the published studies carried out in other ethnic groups. Over the last few years, multiple studies have shown an enhanced risk of recurrent coronary events with high hsCRP levels in normal healthy populations and also people within the various spectra of ischemic heart disease from sub clinical disease through stable angina to acute coronary syndromes in the presence and absence of other cardiovascular risk factors $[6,10,12,26-28,31-35]$.

Our findings indicate that in the Asian Indian population, individuals in the top quartile of hsCRP have an approximately threefold higher risk of reinfarction, even after adjustment for traditional CAD risk factors thus implying that hsCRP may have additional utility for effective risk stratification in this high risk ethnic population. This may strengthen the case towards a recommendation of routine measurement of hsCRP along with lipids for improving risk prediction and identification of high-risk individuals. The AHA-CDC has devised guidelines for the classification of subjects as mild, intermediate and, high risk based on hsCRP levels. However, this classification is based on data obtained 
in Caucasian populations, and there is significant variation in hsCRP level based on ethnic origin [43, 44, 49-52]. Highsensitivity CRP levels are highest among blacks, followed by South Asians, Mexicans, Hispanics, and Caucasians. In addition, Asian Indians have a higher predisposition to diabetes and CVD, resulting in an inherent proinflammatory state [46]. Taking all of these facts into account, the feasibility of application of the AHA-CDC guidelines for classification of subjects needs to be tested before implementation in any population. Upon classifying our study population according to the AHA-CDC guidelines, we found no significant difference in the distribution of CAD affected and unaffected subjects across the three AHA-CDC groups (Figure 1). The unaffected subjects were distributed equally among the three groups. This supports the fact that population-specific reference intervals are required for accurate risk stratification.

\section{Conclusion}

Our data indicate that high levels of hsCRP provide additional predictive value to the traditional risk factors in identififying CAD-affected subjects who could develop a recurrent coronary event. Hence, it appears that routine measurement of hsCRP levels may provide better stratification of CAD risk in this population. However, it is important to carry out large-scale population-based studies in order to modify and adapt the existing AHA-CDC guidelines so as to accurately specify CAD risk in various ethnic populations. None

\section{Author Contribution}

V. S. Rao: concept, planning, study and assay design, data analysis and interpretation, study management and manuscript composition; N. B. Kadarinarasimhiah: biomarker assays; S. John and S. Hebbagodi: data analysis, J. Shanker: study management and manuscript revision V. V. Kakkar: concept, manuscript revision and overall guidance.

\section{Acknowledgments}

The authors gratefully acknowledge the support from Sir Dorabji Tata Trust and also the Thrombosis Research Institute, London, for the IARS. The authors would like to sincerely thank all the subjects who participated in this study. The efforts of the clinical team at the Thrombosis Research Institute, Bangalore, towards enrolment of the study participants and the biostatistics team for maintaining the IARS database are duly acknowledged.

\section{References}

[1] R. Ross, "Atherosclerosis-an inflammatory disease," New England Journal of Medicine, vol. 340, no. 2, pp. 115-126, 1999.

[2] P. Libby, P. M. Ridker, and A. Maseri, "Inflammation and atherosclerosis," Circulation, vol. 105, no. 9, pp. 1135-1143, 2002.

[3] P. Libby, "Inflammation and cardiovascular disease mechanisms," American Journal of Clinical Nutrition, vol. 83, no. 2, pp. 456S-460S, 2006.
[4] R. R. S. Packard and P. Libby, "Inflammation in atherosclerosis: from vascular biology to biomarker discovery and risk prediction," Clinical Chemistry, vol. 54, no. 1, pp. 24-38, 2008.

[5] T. B. Harris, L. Ferrucci, R. P. Tracy et al., "Associations of elevated interleukin- 6 and C-reactive protein levels with mortality in the elderly," American Journal of Medicine, vol. 106, no. 5, pp. 506-512, 1999.

[6] P. M. Ridker, C. H. Hennekens, J. E. Buring, and N. Rifai, "C-reactive protein and other markers of inflammation in the prediction of cardiovascular disease in women," New England Journal of Medicine, vol. 342, no. 12, pp. 836-843, 2000.

[7] P. M. Ridker, N. Rifai, M. Pfeffer, F. Sacks, S. Lepage, and E. Braunwald, "Elevation of tumor necrosis factor- $\alpha$ and increased risk of recurrent coronary events after myocardial infarction," Circulation, vol. 101, no. 18, pp. 2149-2153, 2000.

[8] P. M. Ridker, N. Rifai, M. J. Stampfer, and C. H. Hennekens, "Plasma concentration of interleukin- 6 and the risk of future myocardial infarction among apparently healthy men," Circulation, vol. 101, no. 15, pp. 1767-1772, 2000.

[9] P. Libby and P. M. Ridker, "Inflammation and atherosclerosis: role of C-reactive protein in risk assessment," American Journal of Medicine, vol. 116, no. 6, pp. 9-16, 2004.

[10] W. Koenig, H. Löwel, J. Baumert, and C. Meisinger, "Creactive protein modulates risk prediction based on the Framingham Score implications for future risk assessment: results from a large cohort study in Southern Germany," Circulation, vol. 109, no. 11, pp. 1349-1353, 2004.

[11] M. Cushman, A. M. Arnold, B. M. Psaty et al., "C-reactive protein and the 10-year incidence of coronary heart disease in older men and women: the cardiovascular health study," Circulation, vol. 112, no. 1, pp. 25-31, 2005.

[12] P. M. Ridker, N. Rifai, L. Rose, J. E. Buring, and N. R. Cook, "Comparison of C-reactive protein and low-density lipoprotein cholesterol levels in the prediction of first cardiovascular events," New England Journal of Medicine, vol. 347, no. 20, pp. 1557-1565, 2002.

[13] S. D. de Ferranti and N. Rifai, "C-reactive protein: a nontraditional serum marker of cardiovascular risk," Cardiovascular Pathology, vol. 16, no. 1, pp. 14-21, 2007.

[14] A. W. Taylor, N.-O. Ku, and R. F. Mortensen, "Regulation of cytokine-induced human C-reactive protein production by transforming growth factor- $\beta$," Journal of Immunology, vol. 145, no. 8, pp. 2507-2513, 1990.

[15] Q. Dong and J. R. Wright, "Expression of C-reactive protein by alveolar macrophages," Journal of Immunology, vol. 156, no. 12, pp. 4815-4820, 1996.

[16] Y. X. Zhang, W. J. Cliff, G. I. Schoefl, and G. Higgins, "Coronary C-reactive protein distribution: its relation to development of atherosclerosis," Atherosclerosis, vol. 145, no. 2, pp. 375-379, 1999.

[17] S. Kobayashi, N. Inoue, Y. Ohashi et al., "Interaction of oxidative stress and inflammatory response in coronary plaque instability: important role of C-reactive protein," Arteriosclerosis, Thrombosis, and Vascular Biology, vol. 23, no. 8, pp. 13981404, 2003.

[18] T. Ishikawa, T. Imamura, K. Hatakeyama et al., "Possible contribution of C-reactive protein within coronary plaque to increasing its own plasma levels across coronary circulation," American Journal of Cardiology, vol. 93, no. 5, pp. 611-614, 2004.

[19] M. B. Pepys and G. M. Hirschfield, "C-reactive protein: a critical update," Journal of Clinical Investigation, vol. 111, no. 12, pp. 1805-1812, 2003. 
[20] S. Black, I. Kushner, and D. Samols, "C-reactive protein," Journal of Biological Chemistry, vol. 279, no. 47, pp. 4848748490, 2004.

[21] H. Sun, T. Koike, T. Ichikawa et al., "C-reactive protein in atherosclerotic lesions: its origin and pathophysiological significance," American Journal of Pathology, vol. 167, no. 4, pp. 1139-1148, 2005.

[22] G. J. Blake and P. M. Ridker, "C-reactive protein and other inflammatory risk markers in acute coronary syndromes," Journal of the American College of Cardiology, vol. 41, no. 4 supplement S, pp. 37S-42S, 2003.

[23] P. M. Ridker, S. S. Bassuk, and P. P. Toth, "C-reactive protein and risk of cardiovascular disease: evidence and clinical application," Current Atherosclerosis Reports, vol. 5, no. 5, pp. 341-349, 2003.

[24] P. M. Ridker, M. Cushman, M. J. Stampfer, R. P. Tracy, and C. H. Hennekens, "Inflammation, aspirin, and the risk of cardiovascular disease in apparently healthy men," New England Journal of Medicine, vol. 336, no. 14, pp. 973-979, 1997.

[25] P. M. Ridker, R. J. Glynn, and C. H. Hennekens, "C-reactive protein adds to the predictive value of total and HDL cholesterol in determining risk of first myocardial infarction," Circulation, vol. 97, no. 20, pp. 2007-2011, 1998.

[26] J. Danesh, J. G. Wheeler, G. M. Hirschfield et al., "C-reactive protein and other circulating markers of inflammation in the prediction of coronary heart disease," New England Journal of Medicine, vol. 350, no. 14, pp. 1387-1397, 2004.

[27] M. Cushman, A. M. Arnold, B. M. Psaty et al., "C-reactive protein and the 10-year incidence of coronary heart disease in older men and women: the cardiovascular health study," Circulation, vol. 112, no. 1, pp. 25-31, 2005.

[28] A. Khera, J. A. de Lemos, R. M. Peshock et al., "Relationship between C-reactive protein and subclinical atherosclerosis: the Dallas Heart Study," Circulation, vol. 113, no. 1, pp. 38-43, 2006.

[29] S. M. Boekholdt, C. E. Hack, M. S. Sandhu et al., "C-reactive protein levels and coronary artery disease incidence and mortality in apparently healthy men and women: the EPICNorfolk prospective population study 1993-2003," Atherosclerosis, vol. 187, no. 2, pp. 415-422, 2006.

[30] I. M. van der Meer, M. P. M. de Maat, A. J. Kiliaan, D. A. M. van der Kuip, A. Hofman, and J. C. M. Witteman, "The value of C-reactive protein in cardiovascular risk prediction: the Rotterdam Study," Archives of Internal Medicine, vol. 163, no. 11, pp. 1323-1328, 2003.

[31] A. R. Folsom, L. E. Chambless, C. M. Ballantyne et al., "An assessment of incremental coronary risk prediction using Creactive protein and other novel risk markers: the atherosclerosis risk in communities study," Archives of Internal Medicine, vol. 166, no. 13, pp. 1368-1373, 2006.

[32] W. Koenig, M. Sund, M. Fröhlich et al., "C-reactive protein, a sensitive marker of inflammation, predicts future risk of coronary heart disease in initially healthy middle-aged men: results from the MONICA (monitoring trends and determinants in cardiovascular disease) Augsburg cohort study, 1984 to 1992," Circulation, vol. 99, no. 2, pp. 237-242, 1999.

[33] J. K. Pai, T. Pischon, J. Ma et al., "Inflammatory markers and the risk of coronary heart disease in men and women," New England Journal of Medicine, vol. 351, no. 25, pp. 2599-2610, 2004.

[34] F. Haverkate, S. G. Thompson, S. D. M. Pyke, J. R. Gallimore, and M. B. Pepys, "Production of C-reactive protein and risk of coronary events in stable and unstable angina. European
Concerted Action on Thrombosis and Disabilities Angina Pectoris Study Group," Lancet, vol. 349, no. 9050, pp. 462-466, 1997.

[35] D. E. Laaksonen, L. Niskanen, K. Nyyssönen, K. Punnonen, T.-P. Tuomainen, and J. T. Salonen, "C-reactive protein in the prediction of cardiovascular and overall mortality in middleaged men: a population-based cohort study," European Heart Journal, vol. 26, no. 17, pp. 1783-1789, 2005.

[36] T. A. Pearson, S. N. Blair, S. R. Daniels et al., "AHA Guidelines for Primary Prevention of Cardiovascular Disease and Stroke: 2002 Update: consensus panel guide to comprehensive risk reduction for adult patients without coronary or other atherosclerotic vascular diseases. American Heart Association Science Advisory and Coordinating Committee," Circulation, vol. 106, no. 3, pp. 388-391, 2002.

[37] M. J. Stampfer, P. M. Ridker, and V. J. Dzau, "Risk factor criteria," Circulation, vol. 109, no. 25, pp. V-3-V-5, 2004.

[38] D. Park, P. Desai, J. Aiyengar, and A. Balladur, "Geographic differences in the characteristics of coronary artery disease in India," International Journal of Cardiology, vol. 67, no. 2, pp. 187-189, 1998.

[39] V. Mohan, R. Deepa, S. Shanthi Rani, and G. Premalatha, "Prevalence of coronary artery disease and its relationship to lipids in a selected population in South India: the Chennai Urban Population Study (CUPS no. 5)," Journal of the American College of Cardiology, vol. 38, no. 3, pp. 682-687, 2001.

[40] R. Balarajan, "Ethnicity and variations in mortality from coronary heart disease," Health Trends, vol. 28, pp. 45-51, 1996.

[41] P. M. McKeigue, B. Shah, and M. G. Marmot, "Relation of central obesity and insulin resistance with high diabetes prevalence and cardiovascular risk in South Asians," Lancet, vol. 337, no. 8738, pp. 382-386, 1991.

[42] J. C. Chambers, S. Eda, P. Bassett et al., "C-reactive protein, insulin resistance, central obesity, and coronary heart disease risk in Indian Asians from the United Kingdom compared with European whites," Circulation, vol. 104, no. 2, pp. 145150, 2001.

[43] N. G. Forouhi, N. Sattar, and P. M. McKeigue, "Relation of C-reactive protein to body fat distribution and features of the metabolic syndrome in Europeans and South Asians," International Journal of Obesity, vol. 25, no. 9, pp. 1327-1331, 2001.

[44] E. A. Enas, V. Mohan, M. Deepa, S. Farooq, S. Pazhoor, and H. Chennikkara, "The metabolic syndrome and dyslipidemia among Asian Indians: a population with high rates of diabetes and premature coronary artery disease," Journal of the Cardiometabolic Syndrome, vol. 2, no. 4, pp. 267-275, 2007.

[45] K. Chatha, N. R. Anderson, and R. Gama, "Ethnic variation in C-reactive protein: UK resident Indo-Asians compared with Caucasians," Journal of Cardiovascular Risk, vol. 9, no. 3, pp. 139-141, 2002.

[46] M. Chandalia, A. V. Cabo-Chan Jr., S. Devaraj, I. Jialal, S. M. Grundy, and N. Abate, "Elevated plasma high-sensitivity C-reactive protein concentrations in Asian Indians living in the United States," Journal of Clinical Endocrinology and Metabolism, vol. 88, no. 8, pp. 3773-3776, 2003.

[47] V. Mohan, R. Deepa, K. Velmurugan, and G. Premalatha, "Association of C-reactive protein with body fat, diabetes and coronary artery disease in Asian Indians: the Chennai Urban Rural Epidemiology Study (CURES-6)," Diabetic Medicine, vol. 22, no. 7, pp. 863-870, 2005. 
[48] T. Nyandak, A. Gogna, S. Bansal, and M. Deb, "High sensitive C-reactive protein (hs-CRP) and its correlation with angiographic severity of coronary artery disease (CAD)," Journal, Indian Academy of Clinical Medicine, vol. 8, no. 3, pp. 217-221, 2007.

[49] M. A. Albert, R. J. Glynn, J. Buring, and P. M. Ridker, "Creactive protein levels among women of various ethnic groups living in the United States (from the Women's Health Study)," American Journal of Cardiology, vol. 93, no. 10, pp. 1238-1242, 2004.

[50] A. Khera, D. K. McGuire, S. A. Murphy et al., "Race and gender differences in C-reactive protein levels," Journal of the American College of Cardiology, vol. 46, no. 3, pp. 464-469, 2005.

[51] S. S. Anand, F. Razak, Q. Yi et al., "C-reactive protein as a screening test for cardiovascular risk in a multiethnic population," Arteriosclerosis, Thrombosis, and Vascular Biology, vol. 24, no. 8, pp. 1509-1515, 2004.

[52] E. S. Ford, W. H. Giles, A. H. Mokdad, and G. L. Myers, "Distribution and correlates of C-reactive protein concentrations among adult US women," Clinical Chemistry, vol. 50, no. 3, pp. 574-581, 2004. 


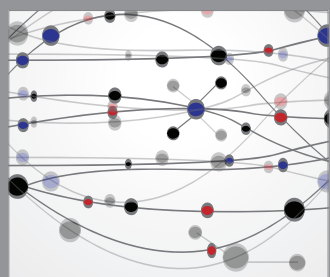

The Scientific World Journal
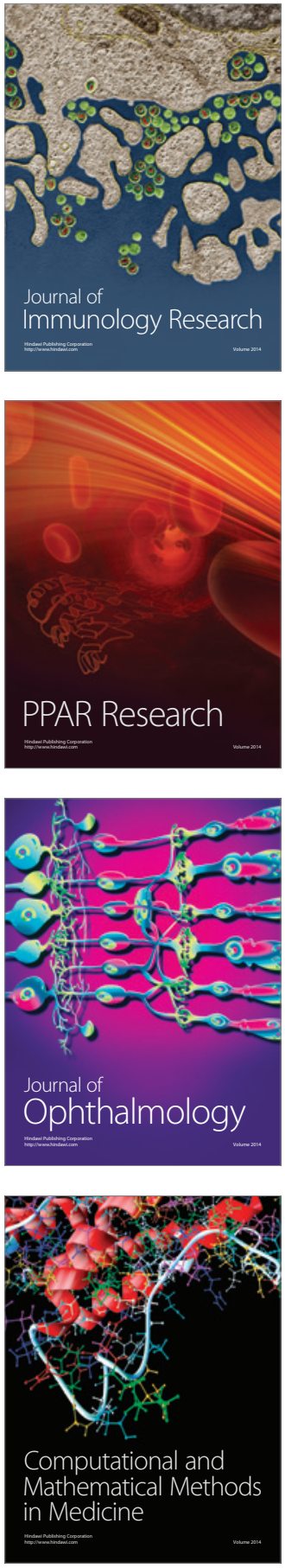

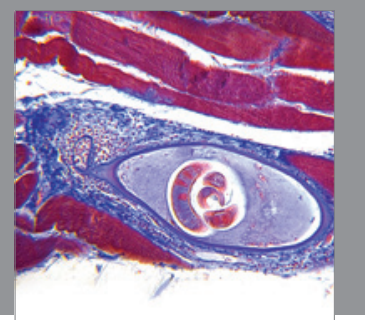

Gastroenterology

Research and Practice
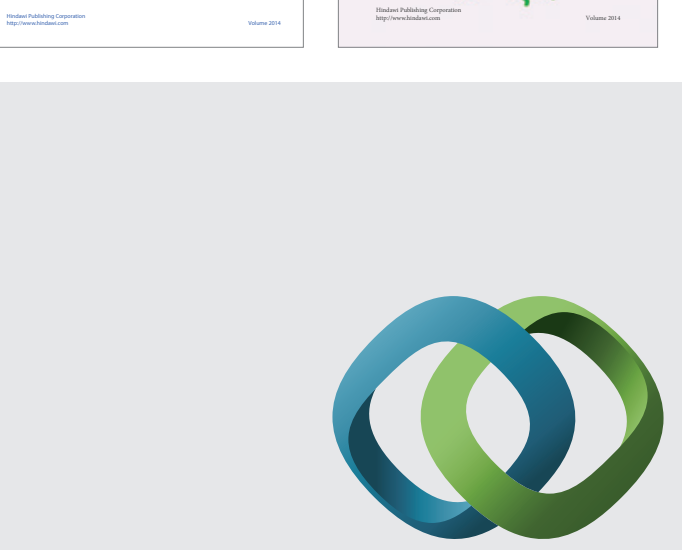

\section{Hindawi}

Submit your manuscripts at

http://www.hindawi.com
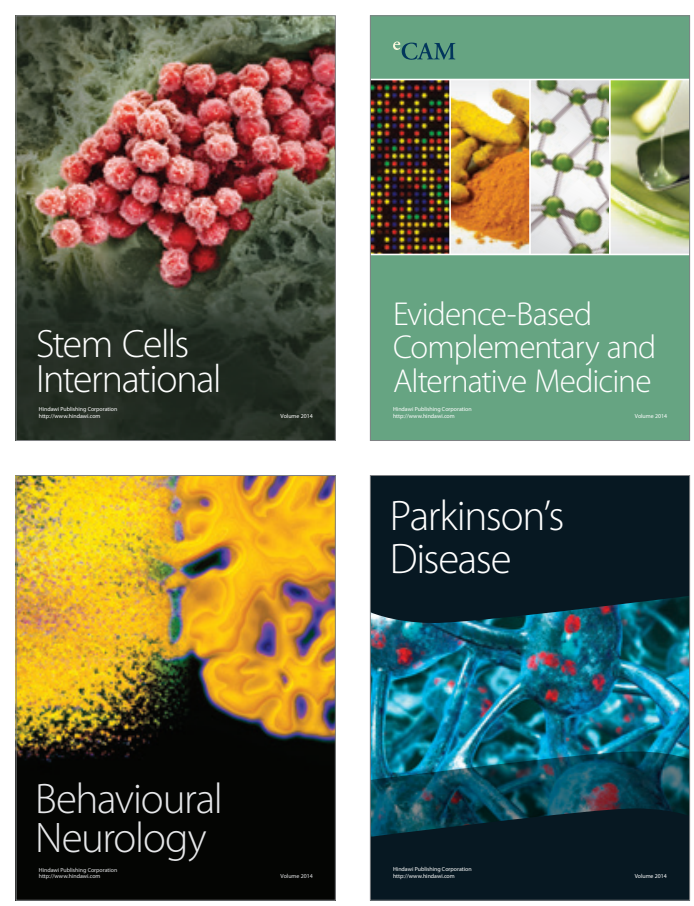

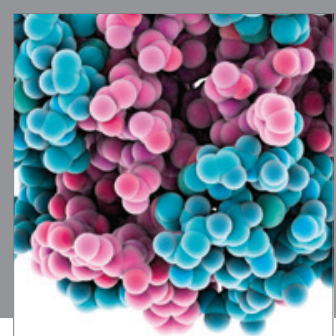

Journal of
Diabetes Research

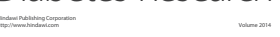

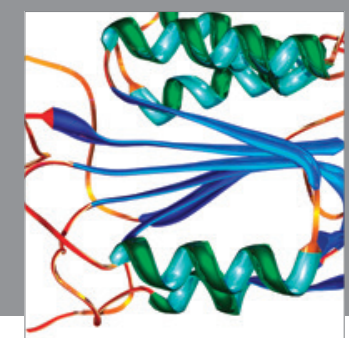

Disease Markers
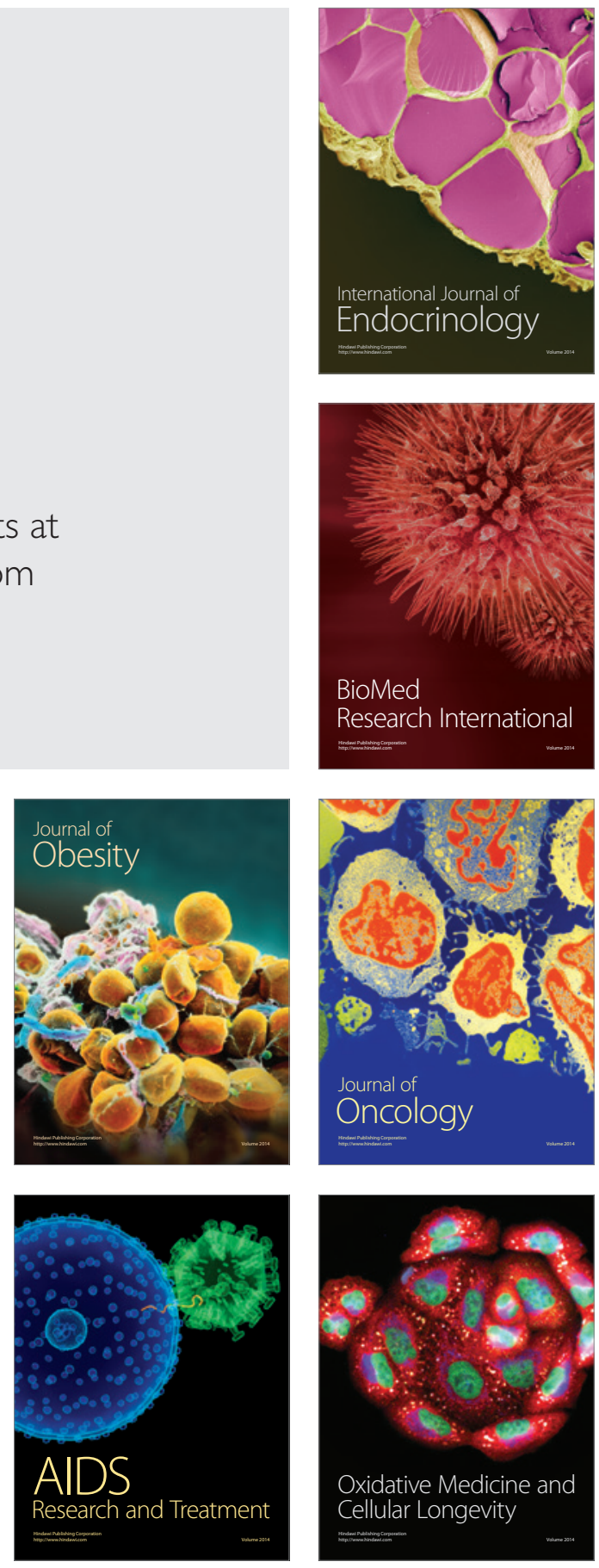